\title{
ACTIVE FAULT DIAGNOSIS IN CLOSED-LOOP SYSTEMS
}

\author{
Henrik Niemann* Niels Kjølstad Poulsen ${ }^{* * 1}$ \\ * Ørsted•DTU, Automation, Technical University of Denmark, \\ Building 326, DK-2800 Kgs. Lyngby, Denmark. \\ E-mail: hhneoersted.dtu.dk \\ ** Informatics and Mathematical Modelling, Technical \\ University of Denmark, \\ Building 321, DK-2800 Kgs. Lyngby, Denmark. \\ E-mail: nkpeimm.dtu.dk
}

\begin{abstract}
Active fault diagnosis (AFD) of parametric faults is considered in connection with closed loop feedback systems. AFD involves auxiliary signals applied on the closed loop system. A fault signature matrix is introduced in connection with AFD and it is shown that if a limited number of faults can occur in the system, a fault separation in the fault signature matrix can be obtained. Then the single elements in the matrix only depend of a reduced number of parametric faults. This can directly be applied for fault isolation. If it is not possible to obtain this separation, it is shown how the fault signature matrix can be applied for a dynamical fault isolation, i.e. fault isolation based on the dynamic characteristic of the fault signature matrix as function of the different parametric faults. Copyright $^{\mathfrak{C}} 2005$ IFAC.
\end{abstract}

Keywords: Active fault diagnosis, parametric faults, passive fault diagnosis, fault signature matrix, closed-loop systems.

\section{INTRODUCTION}

The area of AFD (Active Fault Diagnosis) has obtained an increasing interest in recent years, see e.g. (Campbell and Nikoukhah, 2004; Nikoukhah et al., 2000) and the reference herein. AFD can result in a fast fault detection and/or isolation. It is related with system identification and design of experiments in which auxiliary signals are used to excitate the system.

In the passive fault diagnosis approach, (Frank and Ding, 1994; Gertler, 1998), the diagnosis is only based on existing signals in the system, the control input, the disturbance and the measurement output. As a consequence of this, parametric faults in the system will only be detected/isolated when it is excitated by either

\footnotetext{
1 Corresponding author
}

the control input or the disturbance. It is clear that this will not in general give an optimal fault diagnosis of the system. Instead, if the system is excitated by auxiliary signals, the detection and isolation of parametric faults can be done in a much more systematic way. In many cases, the fault diagnosis will also be much faster.

The AFD also has drawbacks. The system is excitated by auxiliary signals. These signals might disturb the performance of the system in the fault free case. Therefore, the auxiliary input needs to be designed such that the effect on the performance is minimized, but it should still be possible to detect/isolate parametric faults in the system.

The main focus in this paper is on the setup of active fault detection in closed loop system. The fault sig- 
nature matrix will be introduced as a central transfer function in connection with AFD. It will be shown that the fault signature matrix in some cases can be applied for direct fault isolation. If a direct fault isolation cannot be obtained, a dynamic fault isolation needs to be done. A design problem with respect to optimize the auxiliary input signals for obtaining dynamic fault isolation is formulated. An example study is included to verify and illustrate the results.

\section{SYSTEM SETUP}

Consider the following generalized nominal system:

$$
\Sigma_{P}:\left\{\begin{array}{l}
z=G_{z w} w+G_{z d} d+G_{z u} u \\
e=G_{e w} w+G_{e d} d+G_{e u} u \\
y=G_{y w} w+G_{y d} d+G_{y u} u
\end{array}\right.
$$

where $d \in \mathcal{R}^{r}$ is a disturbance signal vector, $u \in \mathcal{R}^{m}$ the control input signal vector, $e \in R^{q}$ is the external output signal vector to be controlled, $y \in \mathcal{R}^{p}$ is the measurement vector, $w \in \mathcal{R}^{k}$ and $z \in \mathcal{R}^{k}$ are external input and output vectors. The connection between the external output $z$ and the external input $w$ is given by

$$
w=\theta z
$$

where $\theta$ is a diagonal matrix

$$
\theta=\operatorname{diag}\left(\theta_{1}, \cdots, \theta_{i}, \cdots, \theta_{k}\right)
$$

representing the parametric faults in the system. We will use the notation $\theta_{i} \neq 0$ as a short form for

$$
\theta=\operatorname{diag}\left(0, \cdots, 0, \theta_{i}, 0, \cdots, 0\right)
$$

i.e. $\theta_{j}=0, j \neq i$. Note that the above description is also applied in connection with the description of systems including model uncertainties. Closing the loop from $w$ to $z$ in $\Sigma_{P}$ by using $\theta$, we get

$$
\Sigma_{P, \theta}=\mathcal{F}_{u}\left(\Sigma_{P}, \theta\right)
$$

$\Sigma_{P, \theta}$ is given by

$$
\Sigma_{P, \theta}:\left\{\begin{array}{l}
e=G_{e d}(\theta) d+G_{e u}(\theta) u \\
y=G_{y d}(\theta) d+G_{y u}(\theta) u
\end{array}\right.
$$

Further, let the system be controlled by a stabilizing feedback controller given by:

$$
\Sigma_{K}:\{u=K y
$$

\subsection{Coprime factorization}

Let a coprime factorization of the system $G_{y u}(s)$ from (1) and a stabilizing controller $K(s)$ from (3) be given by:

$$
\begin{aligned}
& G_{y u}=N M^{-1}=\tilde{M}^{-1} \tilde{N}, N, M, \tilde{N}, \tilde{M} \in \mathcal{R} \mathcal{H}_{\infty} \\
& K \quad=U V^{-1}=\tilde{V}^{-1} \tilde{U}, \quad U, V, \tilde{U}, \tilde{V} \in \mathcal{R} \mathcal{H}_{\infty}
\end{aligned}
$$

where the eight matrices in (4) must satisfy the double Bezout equation given by, see (Tay et al., 1997):

$$
\begin{aligned}
\left(\begin{array}{ll}
I & 0 \\
0 & I
\end{array}\right) & =\left(\begin{array}{cc}
\tilde{V} & -\tilde{U} \\
-\tilde{N} & \tilde{M}
\end{array}\right)\left(\begin{array}{cc}
M & U \\
N & V
\end{array}\right) \\
& =\left(\begin{array}{cc}
M & U \\
N & V
\end{array}\right)\left(\begin{array}{cc}
\tilde{V} & -\tilde{U} \\
-\tilde{N} & \tilde{M}
\end{array}\right)
\end{aligned}
$$

\subsection{Feedback Control}

Based on the system $\Sigma_{P, \theta}$ given by (2) and the feedback controller $\Sigma_{K}$ given by (3), the following block diagram shown in Fig. 1 can be derived.

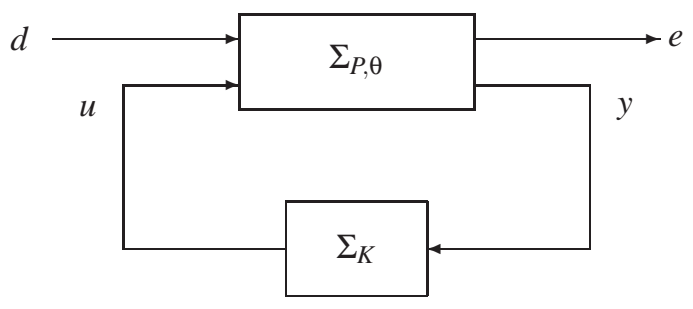

Fig. 1. Feedback control system.

Now, including also a residual vector in connection with the feedback control system in Fig. 1. A residual vector $r$ can be given by, (Frank and Ding, 1994)

$$
r=\tilde{M} y-\tilde{N} u
$$

Notice, the relation to the innovation signal in an ARX structure and system identification.

Let's use the feedback controller described by coprime factors, i.e. $K=\tilde{V}^{-1} \tilde{U}$, in the block diagram. Further, include also an auxiliary input vector $\eta$ in between the two controller coprime matrices in feedback controller. The block diagram in Fig. 1 including $\eta$ and $r$ is shown in Fig. 2. The two signal vectors, $\eta$ and $r$, will be applied in connection with the active fault diagnosis.

Based on the feedback system in Fig. 2, it is possible to give the transfer functions from the two input vectors $d, \eta$ to the two output vectors $e, r$. This is given by:

$$
\left(\begin{array}{l}
e \\
r
\end{array}\right)=\left(\begin{array}{ll}
P_{e d} & P_{e \eta} \\
P_{r d} & P_{r \eta}
\end{array}\right)\left(\begin{array}{l}
d \\
\eta
\end{array}\right)
$$

where $P$ is given by (Niemann and Stoustrup, 2002):

$$
\begin{aligned}
P_{e d} & =G_{e d}(\theta)+G_{e u}(\theta) U\left(V-G_{y u}(\theta) U\right)^{-1} G_{y d}(\theta) \\
P_{e \eta} & =G_{e u}(\theta)\left(M-U\left(V-G_{y u}(\theta) U\right)^{-1}\left(N-G_{y u}(\theta) M\right)\right) \\
P_{r d} & =\left(V-G_{y u}(\theta) U\right)^{-1} G_{y d}(\theta) \\
P_{r \eta} & =-\left(V-G_{y u}(\theta) U\right)^{-1}\left(N-G_{y u}(\theta) M\right)
\end{aligned}
$$

Note that the transfer function from the input vector $\eta$ to the residual vector $r$ is equal to the dual YJBK parameter, (Niemann, 2003; Tay et al., 1997). The dual YJBK parameterization gives the parameterization of all systems stabilized by a fixed feedback controller 


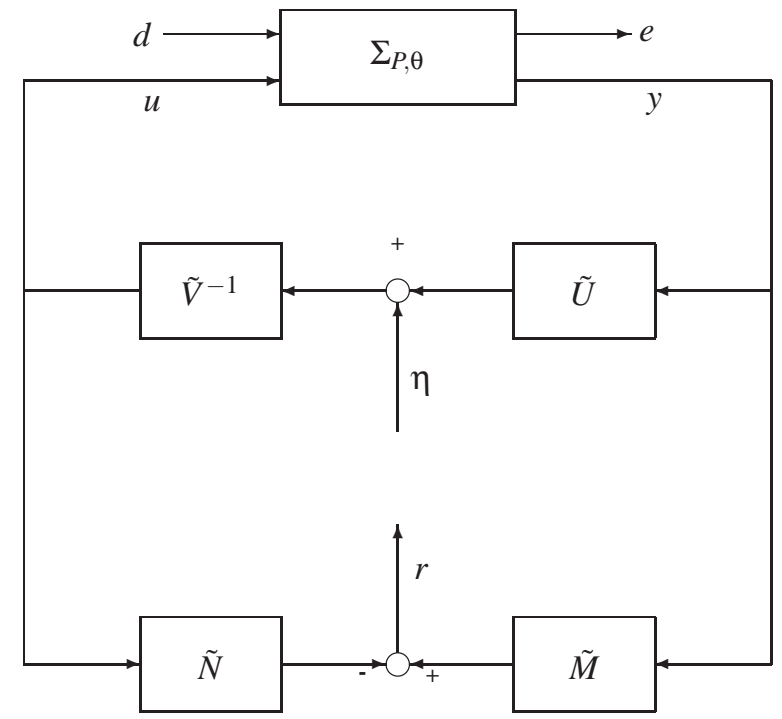

Fig. 2. Controller structure including residual vector $r$ and the external input vector $\eta$.

in terms of the stable dual YJBK parameter. The dual YJBK parameter is normally denoted $S$. We will use $S$ instead of $P_{r \eta}$ in the rest of this paper.

It is possible to rewrite $S=P_{r \eta}$ given by (8) into, (Niemann, 2003):

$$
S(\theta)=\tilde{M} G_{y w} \theta\left(I-\left(G_{z w}+G_{z u} U \tilde{M} G_{y w}\right) \theta\right)^{-1} G_{z u} M
$$

(9) gives a direct description of the parametric faults effect on the closed loop stability. If $S$ gets unstable for some faults, the closed loop system will be unstable. A large $S$ indicates that the faults has a major effect on the system. A large $S$ will however also facilitate the ability to detect the fault. Further, $S$ also shows the equivalence between parametric faults and system uncertainty. This equivalence is very useful in obtaining systematic methods for fault tolerant control, see e.g. (Niemann and Stoustrup, 2002).

\section{PASSIVE FAULT DIAGNOSIS}

It has been shown in (Frank and Ding, 1994) that it is possible to parameterize all residual generators by using the YJBK parameterization. All residual vectors $r_{q}$ for the $\Sigma_{P}$ given by (1) can be described by

$$
r_{q}=Q_{\mathrm{FDI}, O}(\tilde{M} y-\tilde{N} u)=Q_{\mathrm{FDI}, o} r
$$

where $Q_{\mathrm{FDI}, O}$ is a stable and proper filter of suitable order. $Q_{\mathrm{FDI}, O}$ needs to be designed such that the residual signal/vector $r_{q}$ satisfies the following conditions, (Saberi et al., 2000):

- Fault detection

$$
\begin{aligned}
& r_{q}(t)=0 \text { for } \theta=0, \forall(d(t), u(t)) \\
& r_{q}(t) \neq 0 \text { for } \theta \neq 0, \forall(d(t), u(t)) \neq(0,0)
\end{aligned}
$$

- Fault isolation

$$
\begin{aligned}
& r_{q}(t)=0 \text { for } \theta=0, \forall(d(t), u(t)) \\
& r_{q, i}(t) \neq 0 \text { for } \theta_{i} \neq 0, \forall(d(t), u(t)) \neq(0,0) \\
& r_{q, j}(t)=0 \text { for } \theta_{j}=0, j \neq i, \forall(d(t), u(t))
\end{aligned}
$$

Note that the definitions of fault detection and isolation given above are not unique. Depending on how many faults that can occur simultaneously, different definitions can be given, (Saberi et al., 2000). It is also important to point out that it is not always possible to design $Q_{\mathrm{FDI}, O}$ such that it is possible to obtain exact fault detection and fault isolation. Instead, different forms of approximative fault detection and/or fault isolation need to be considered, (Frank and Ding, 1994).

\section{ACTIVE FAULT DIAGNOSIS}

The system given by (7) will now be applied in connection with AFD. Let the system be given by:

$$
\Sigma_{\mathrm{FDI}}:\left\{\begin{array}{l}
e=P_{e d}(\theta) d+P_{e \eta}(\theta) \eta \\
r=P_{r d}(\theta) d+S(\theta) \eta
\end{array}\right.
$$

where $(d, e)$ are external disturbance input and performance output vectors, respectively, and $(\eta, r)$ are internal auxiliary input and residual output vectors, respectively. The internal vectors will be applied for AFD. The system $\Sigma_{\text {FDI }}$ is shown in Fig. 3.

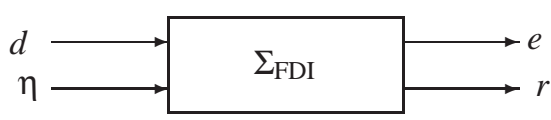

Fig. 3. The system setup for active fault diagnosis.

The first important observation of $\Sigma_{\mathrm{FDI}}$ is that $S(\theta)$ is zero in the fault free case, i.e.

$$
S(0)=0
$$

It is clear from this first observation, that $S(\theta)$ is very important in connection with AFD. Following the definition of fault signature for additive faults in (Massoumnia, 1986), $S(\theta)$ will be named as the fault signature matrix in connection with parametric faults, (Niemann, 2005a; Niemann, 2005b).

\subsection{Direct Fault Diagnosis based on S.}

The definition of fault detection and isolation given in Section 3 in the passive fault diagnosis case takes the following form in the AFD case:

- Fault detection

$$
\begin{aligned}
& S(\theta)=0 \text { for } \theta=0 \\
& S(\theta) \neq 0 \text { for } \theta \neq 0
\end{aligned}
$$

- Fault isolation

$$
\begin{array}{ll}
S(\theta)=0 & \text { for } \theta=0 \\
S(\theta)=S\left(\theta_{i}\right) & \text { for } \theta_{i} \neq 0 \\
S(\theta) \neq S\left(\theta_{j}\right) & \text { for } \theta_{j}=0, j \neq i
\end{array}
$$


It should again be pointed out, that it is possible to give other definitions for fault isolation, depending on the number of faults that can occur simultaneously. In the most common case, it is assumed that only a single fault occurs by itself, i.e. two faults never occur simultaneously. Based on this, it is possible to simplify the fault signature matrix $S(\theta)$ given by (9). Let the closed loop transfer function from $w$ to $z$ be given by

$$
G_{z w, c l}=G_{z w}+G_{z u} U \tilde{M} G_{y w}
$$

Let the $i^{\prime} t h$ fault occur in the system, i.e. $\theta_{i} \neq 0$. The fault signature matrix in (9) can then be written as

$$
S\left(\theta_{i}\right)=\frac{\theta_{i}}{1-\left(G_{z w, c l}\right)_{i i} \theta_{i}} \tilde{M} G_{y w} \Xi_{i i} G_{z u} M
$$

where $\Xi_{i i}$ is a $k \times k$ zero matrix with 1 at element $(i, i)$ and $\left(G_{z w, c l}\right)_{i i}$ element $(i, i)$ of $G_{z w, c l}$.

Observing that both $\tilde{M} G_{y w}$ and $G_{z u} M$ are stable transfer matrices. Furthermore $S\left(\theta_{i}\right)$ in (13) is stable if the SISO transfer function

$$
\frac{\theta_{i}}{1-\left(G_{z w, c l}\right)_{i i} \theta_{i}}
$$

is stable.

It is clear that the rank of the fault signature matrix $S\left(\theta_{i}\right)$ in (13) is 1 . This means that it should be possible to get a reduction of $S\left(\theta_{i}\right)$ by using a pre-filter $Q_{\mathrm{FDI}, I}$ and a post-filter $Q_{\mathrm{FDI}, O}$ around $S\left(\theta_{i}\right) . S\left(\theta_{i}\right)$ is then given by

$$
S_{Q}\left(\theta_{i}\right)=Q_{\mathrm{FDI}, o} \frac{\theta_{i}}{1-\left(G_{z w, c l}\right)_{i i} \theta_{i}} \tilde{M} G_{y w} \Xi_{i i} G_{z u} M Q_{\mathrm{FDI}, I}
$$

If $\tilde{M} G_{y w}$ is left invertible, it is possible to find a stable weight function $Q_{\mathrm{FDI}, O}$ such that $Q_{\mathrm{FDI}, O} \tilde{M} G_{y w}$ is a stable diagonal matrix. This will result in a row separation of the faults in the fault signature matrix. The $i^{\prime}$ th row of $S_{Q}\left(\theta_{i}\right)$ will only depend on the single parametric fault $\theta_{i}$. In the case when $G_{z u} M Q_{\mathrm{FDI}, I}$ is right invertible, a column separation in $S_{Q}\left(\theta_{i}\right)$ can be obtained, see (Niemann, 2005a) for further details.

\subsection{Dynamic Diagnosis}

In the case where a direct fault diagnosis based on the structure of the fault signature matrix $S(\theta)$ can be done, the auxiliary vector signal is only used to detect if $S_{i j}(\theta) \neq 0$ or $S_{i j}(\theta)=0$. If this is not possible, a dynamic fault diagnosis must be done. In this case, the auxiliary vector must be designed with respect to obtain this dynamic diagnosis. An equivalent design problem has been considered in details in (Campbell and Nikoukhah, 2004). In (Campbell and Nikoukhah, 2004), it is assumed that the external output and the measurement output are the same. Using the fault diagnosis setup, based on the fault signature matrix as considered in this paper, results in a more general setup as shown in Fig. 3.
Consider $\Sigma_{\text {FDI }}$ given by (11) in the fault free case, $\theta=0$. The four transfer functions are given by:

$$
\begin{aligned}
P_{e d}(0) & =G_{e d}(0)+G_{e u}(0) U \tilde{M} G_{y d}(0) \\
P_{e \eta}(0) & =G_{e u}(0) M \\
P_{r d}(0) & =\tilde{M} G_{y d}(0) \\
S(0) & =0
\end{aligned}
$$

Let's again assume that only a single parametric fault occurs by itself. This is without loss of generality. We have then the following hypothesis:

$$
\begin{aligned}
H_{0} & : \Sigma_{\mathrm{FDI}}(\theta=0) \\
H_{i} & : \Sigma_{\mathrm{FDI}}\left(\theta=\theta_{i}\right), i=1, \cdots, k
\end{aligned}
$$

where the associated transfer functions are given by (15) for $H_{0}$ (the fault free case) and by (8) and (13) for $H_{i}$.

The auxiliary input signal $\eta$ must be designed with respect to:

- Minimize the effect from $\eta$ on the external output $e$ in the fault free case.

- Maximize the signature from $\eta$ on $r$ in the fault case.

In the case of faults, the degradation of the performance due to the auxiliary input is not important. A degradation of the performance will in general always be the results of faults in the system. It is here more important to detect the faults and subsequently handle the faulty situation in a proper way.

In order to handle both deterministic and stochastic components in the signals let us for the process, $y$, introduce

$$
\bar{V}(y)=\lim _{T \rightarrow \infty} \frac{1}{T} \int_{0}^{T} g\left(\mathbf{E}\left\{y y^{T}\right\}\right) d t
$$

where $y$ is a (possibly generated) stochastic process with properties (second order) so that the function exists. Here $g$ is a scalar valued function (e.g. $\operatorname{det}(\cdot), \operatorname{tr}(\cdot)$ or $g(X)=C X C^{T}$ for a specific row vector, $C)$. Let us for short denote $\bar{V}$ as the variance. A measure of the performace degradation for the nominal system could be

$$
J_{H_{0}}^{s}=\frac{\bar{V}\left(P_{e \eta}(0) \eta\right)}{\bar{V}\left(P_{e d}(0) d\right)}
$$

depending on the overal control objective and its relation to cost function of the type in (16).

A fault detection could be based on a test for variance increment in the residual, if that is the result of a fault. However, a fault could also result in a reduction in variance of the residual. The detection could in both cases be implemented as a CUSUM detector, (Basseville and Nikiforov, 1993).

The chance of detecting a fault by means of passive methods depends on the change in variance i.e. on

$$
\frac{\bar{V}\left(P_{r d}(\theta) d\right)}{\bar{V}\left(P_{e d}(0) d\right)}
$$


If this fraction is close to one the detection might be difficult. In the case of variance increment the problem can be reduced if an active method is used and a stochastic probe (auxiliary) signal, $\eta$, is introduced. For ( $\eta$ independent of $d$ ) the ration becomes

$$
\frac{\bar{V}\left(P_{r d}(\theta) d\right)+\bar{V}(S(\theta) \eta)}{\bar{V}\left(P_{e d}(0) d\right)}
$$

since $\bar{V}(S(0) \eta)=0$. If, on the other hand, the fault results in a decreased variance, then this method seems intractable and is not pursuited further in this paper.

In the following we will focus on a method which is based on the application of a probe signal with a distinct signature in the residual signal. Such a signal could be a harmonic function with angular frequency $\omega_{0}$ and amplitude $A$. The indicator function could be

$$
\underline{V}(y)=\left|\lim _{T \rightarrow \infty} \frac{1}{T} \int_{0}^{T} \mathbf{E}\{y\} e^{j \omega_{0} t} d t\right|
$$

The amplitude has to be determined such that the performance degradation in (17) is limited (to $c$ ). In order to maximize the signature, $\underline{V}(S(\theta) \eta)$, the frequency, $\omega_{0}$, must be found as the maximum to $S(\theta) P_{e \eta}^{-1}(0)$.

\section{EXAMPLE STUDY}

Consider the following first order system:

$$
G_{y u}(s)=\frac{k}{1+\tau s}=\frac{1}{\tau} \frac{k}{s+1 / \tau}
$$

It is assumed that the $k$ and the time constant $\tau$ can be faulty. A parametric fault model is applied given by:

$$
\begin{aligned}
k & =k_{0}\left(1+\theta_{k}\right) \\
\frac{1}{\tau} & =\frac{1}{\tau_{0}}\left(1+\theta_{\tau}\right)
\end{aligned}
$$

where $k_{0}$ and $\tau_{0}$ are the fault free parameters. Using the system description given by (1), $\Sigma_{P}$ is then given by the following state space form:

$$
\Sigma_{P}:\left\{\begin{array}{l}
\dot{x}=-\frac{1}{\tau_{0}} x+\left(k_{0} \frac{-1}{\tau_{0}}\right) w+k_{0} u \\
z=\left(\begin{array}{l}
0 \\
1
\end{array}\right) x+\left(\begin{array}{l}
1 \\
0
\end{array}\right) u \\
y=\frac{1}{\tau_{0}} x+\left(0 \frac{1}{\tau_{0}}\right) w
\end{array}\right.
$$

where the loop from $z$ to $w$ is closed by $\theta$ given by

$$
\theta=\operatorname{diag}\left(\theta_{k}, \theta_{\tau}\right)
$$

Based on the system setup in (21), it is possible to give the equation for the fault signature matrix $S$ as function of $\theta_{k}$ and $\theta_{\tau}$. Assume that an observer based feedback controller is applied. Let $f$ be a stabilizing state feedback gain and $l$ be a stabilizing observer gain, i.e. $\left(-\frac{1}{\tau_{0}}+k_{0} f\right)$ and $\frac{1}{\tau_{0}}(-1+l)$ are stable, respectively. $S$ can then be calculated directly from (9).

In the following $k_{0}=1$ and $\tau_{0}=1$ and the controller is designed with $f=-2$ and $l=-3$. This results in closed loop poles in -3 and -4 for the controller and observer, respectively. The plant is characterized by an input disturbance, i.e. $G_{y d}=G_{y u}$ and the disturbance is modelled as white noise with intensity equal 0.1 . Furthermore is $G_{e u}=G_{e d}=G_{y u}$.

Assume that the single fault results in $k=1.1$ (or $\theta_{k}=$ $\left.0.1, \theta_{\tau}=0\right)$. In Figure 4 the amplitude spectrum for $S(9,0)$ (equivalent to $k=10)$ and $P_{e \eta}(0)$ is depicted. $\theta_{k}=9$ is selected for illustration of the effect from $\theta_{k}$ on $S . \theta_{k}=0.1$ is applied in the rest of the example. Let $\omega_{0}$ be (angular) frequency at which the transfer function $S P_{e \eta}^{-1}$ attain is maximum value. If the task is to maximize the fault signature in $r$ subject to a constrained degradation in $e$ under normal operation (no fault), then the optimal probe signal is a harmonic with frequency $\omega_{0}$. From Fig. 4 is easily seen that $\omega_{0} \simeq 10 \mathrm{~Hz}$. In Fig. 5 it can also be seen that this choice is fairly robust.

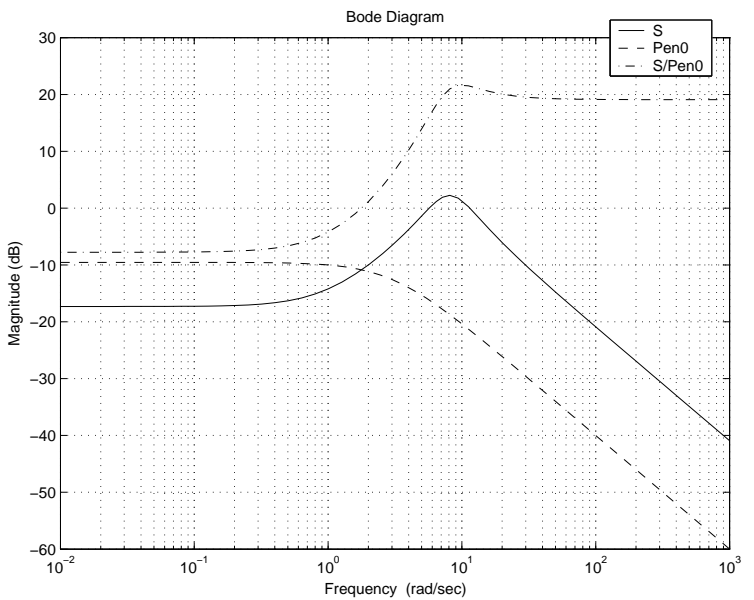

Fig. 4. Bode plot for $k=10$ showing $S(9,0), P_{e \eta}(0)$ and their ratio.

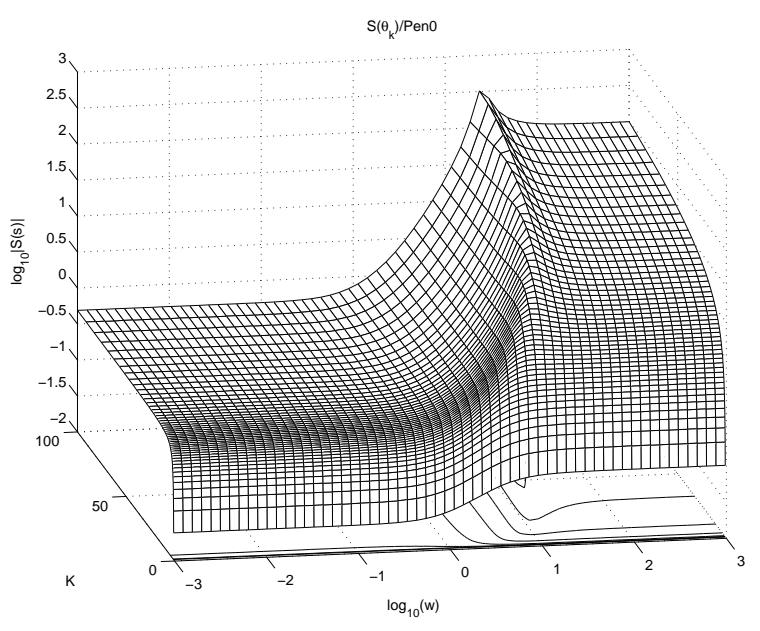

Fig. 5. Bode plot $S\left(\theta_{k}\right) P_{e \eta}^{-1}(0)$ as function of $k$.

The results from a simulation experiment are shown in the Figs. 6, 7 and 8. The fault occurs at $t=10 \mathrm{sec}$ and the probe signal is only active in the period from $5 \mathrm{sec}$ to $15 \mathrm{sec}$. The quality of the control can be measured as the variation in the error signal, $e$ (see 
Fig. 2 and the signal in 6). As a result of $c=0.1$ the effect of the probe signal is barely seen in the error signal before the fault occurs (in the period from $5 \mathrm{sec}$ to $10 \mathrm{sec}$ ). The detection is illustrated by means of the accumualted signal

$$
\delta=\left|\int_{0}^{T} r e^{j \omega_{0} t} d t\right|
$$

in Fig. 8, but a real time implementation should be based on a CUSUM version.

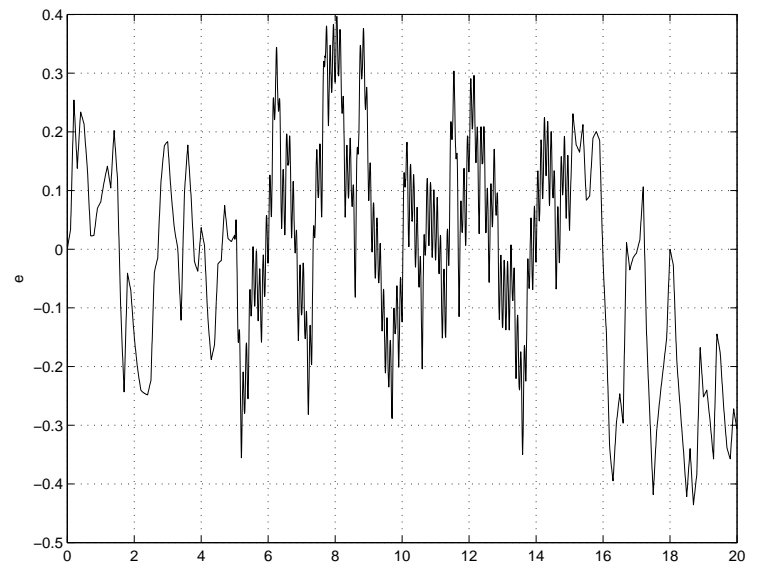

Fig. 6. The error signal, $e$, obtained in the simulation.

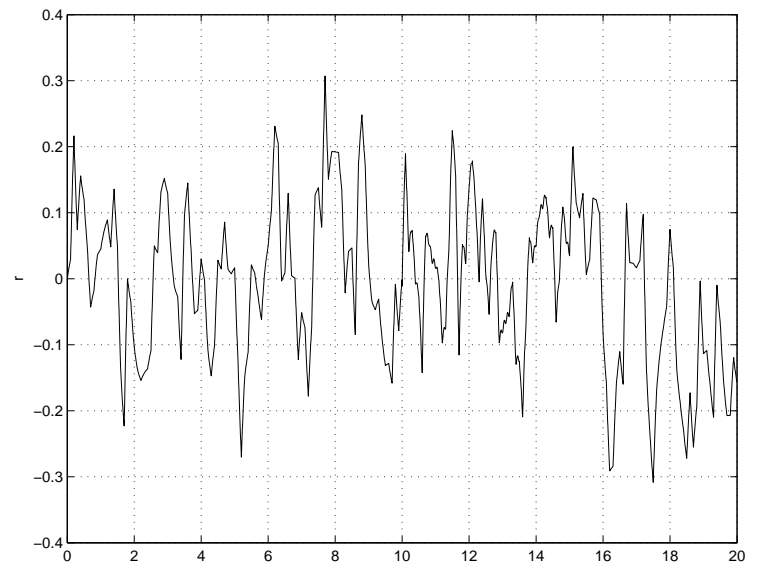

Fig. 7. The residual, $r$, signal obtained in the simulation.

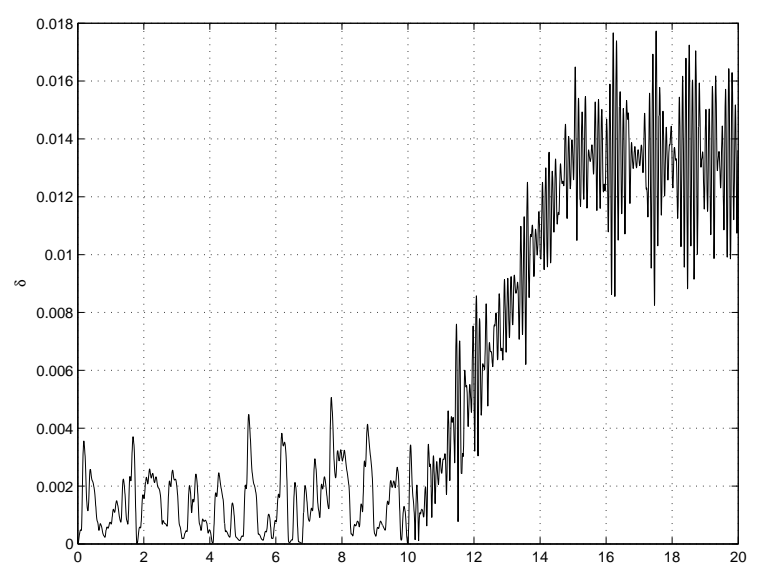

Fig. 8. The $\delta$ signal

\section{CONCLUSION}

Active fault diagnosis has been applied in closedloop systems. A fault signature matrix $S$ has been introduced. It has been shown how the fault signature matrix is central in the AFD. The gain of $S$ is a direct indication of the effect from the faults on the system. Based on this matrix, direct fault detection and fault isolation can be obtained in some cases. In the cases where direct FDI is not possible, dynamic FDI must be carried out. An index for the design of auxiliary input (probe) signals has been formulated. An example shows how it is possible to detect small faults using an auxiliary signal with only a minor performance reduction of the nominal system.

\section{REFERENCES}

Basseville, M. and I.V. Nikiforov (1993). Detection of abrupt changes - theory and application. Prentice Hall.

Campbell, S.L. and R. Nikoukhah (2004). Auxiliary signal design for failure detection. Princeton University Press. Princeton, NJ, USA.

Frank, P.M. and X. Ding (1994). Frequency domain approach to optimally robust residual generation and evaluation for model-based fault diagnosis. Automatica 30, 789-804.

Gertler, J. (1998). Fault detection and diagnosis in engineering systems. Marcel Dekker.

Massoumnia, M. (1986). A geometric approach to the synthesis of failure detection filters. IEEE Transactions on Automatic Control 31(9), 839846.

Niemann, H.H. (2003). Dual Youla parameterization. IEE Proceedings - Control Theory and Applications 150(5), 493-497.

Niemann, H.H. (2005a). Algebraic aspect of active fault diagnosis in closed-loop systems. Submitted for publication, avaible at www.oersted.dtu.dk under "Publications".

Niemann, H.H. (2005b). Fault tolerant control based on active fault diagnosis. In: Proceedings of the American Control Conference. Portland, Oregon, USA. pp. 000-000.

Niemann, H.H. and J. Stoustrup (2002). Reliable control using the primary and dual Youla parameterization. In: Proceedings of the 41st IEEE Conference on Decision and Control. Las Vegas, NV, USA. pp. 4353-4358.

Nikoukhah, R., S.L. Campbell and F. Delebecque (2000). Detection signal design for failure detection: a robust approach. International Journal of Adaptive Control and Signal Processing 14, 701724.

Saberi, A., A.A. Stoorvogel, P. Sannuti and H.H. Niemann (2000). Fundamental problems in fault detection and identification. International Journal of Robust and Nonlinear Control 10(14), 12091236.

Tay, T.T., I.M.Y. Mareels and J.B. Moore (1997). High performance control. Birkhäuser. 